\title{
40th ANNUAL SASKATCHEWAN CHRISTMAS BIRD COUNT - 1981
}

Compiled by MARGARET BELCHER, 2601 Winnipeg Street, Regina, Saskatchewan. S4P $1 \mathrm{H} 8$

In the absence of Mary Houston, who is in Ann Arbor, Michigan, with her husband on his sabbatical leave, I have taken on her onerous task of compling the Saskatchewan Christmas bird counts. During the count period (19 December 1981 to 3 January 1982) $54^{*}$ counts were reported and 82 species of birds were seen, 78 of these being recorded on count day. The highest numbers of species were counted at Fort Walsh (36), Saskatoon (37), and Squaw Rapids (36).

Three species are new to the Saskat-

*55 with the belated receipt of the count from Togo. chewan Christmas Bird Count Double-crested Cormorant, Loggerhead Shrike, and Chipping Sparrow. The Double-crested Cormorant was observed at Squaw Rapids. The Loggerhead Shrike was seen at Fort Walsh by Ron Jensen who provided this description from his field notes: "Uniform grey, with black stripe over bill, hooking down BEYOND eye. Viewed in full sun (front-lit) with $8 \times 40$ binoculars for one minute at 30 feet." The Chipping Sparrow at Indian Head was a single bird that had been coming to Fred Skinner's feeder since the fall; he reported it on count day, but it was not seen after 26 December. With these new species, the all-time Saskat-

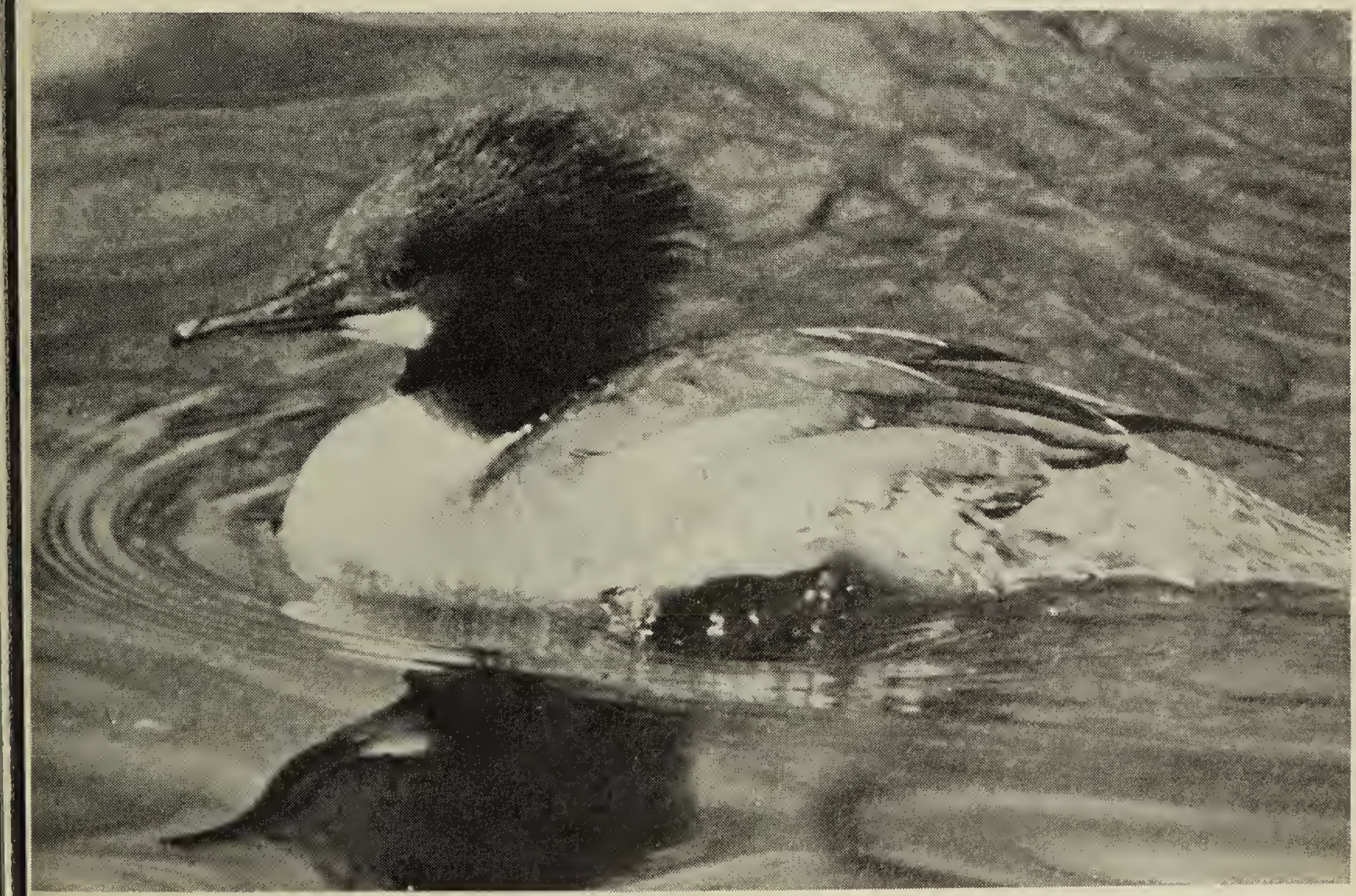


chewan total for species seen on count day is now 133.

Some of the rarities of previous years have reappeared -1 Turkey at Biggar, 3 Common Snipe at Fort Walsh, 1 Northern Cardinal at Saskatoon. Waterfowl are wintering in several localities, their presence explained by special circumstances that create open water. At Killsquaw Lake near Unity, for example, ducks are wintering at the Sifto Salt Plant. At Gardiner Dam, the reservoir was mostly open on 21 December, count day, for only the second time in nine years, and a White Pelican and three Whistling Swans were observed there with several species of ducks. Perhaps the most impressive species count was that of 52 Bald Eagles at Squaw Rapids.

In the list that follows, the names of the observers are given for each locality. Table 1 gives details of count coverage and weather, Table 2 gives the numbers seen for those species reported in more than three localities, while species seen in three or fewer localities are reported in Table 3. Species recorded during the count period, but not on count day, have a plus sign before the number of individuals seen.

In the tables, only birds identified as to species appear. Thus the number of individuals given in the table will be less than the number actually seen on the count if any of those seen were not positively identified. In a few cases, the number of species listed at the bottom of the column will also be less than the number actually seen. For example, at Regina an accipiter (either a Cooper's or Sharp-shinned) and three blackbirds (the only ones seen on the count) would bring the published total of 26 species up to 28. On several counts, there were buteos and small owls not seen well enough to be sure of the species. Information on these, as well as dates of species seen during the count period but not on count day, and descriptions of the count areas, are available in the files.

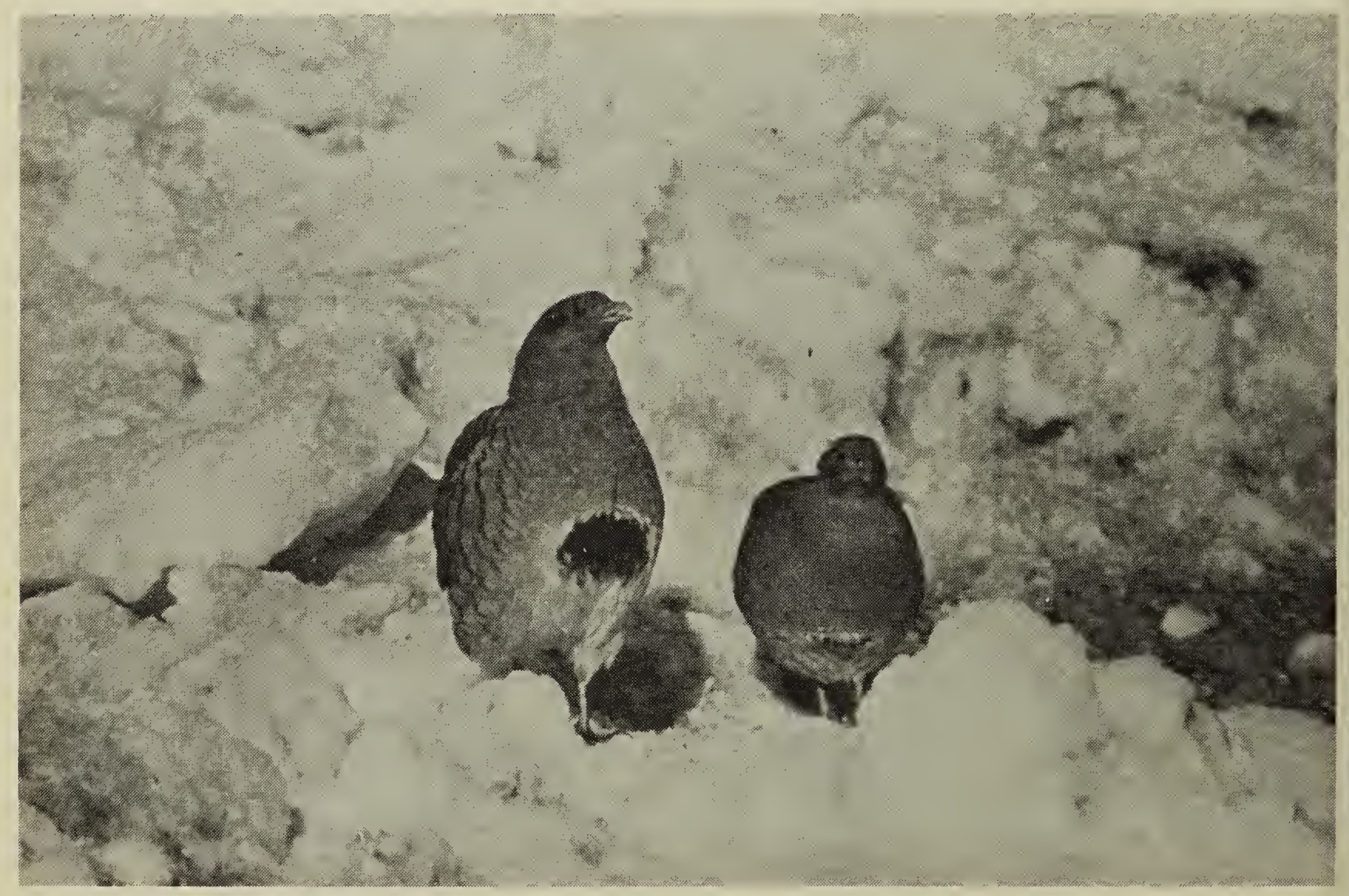


1. ASSINIBOIA

2. BANGOR

3. BIGGAR

4. BIG GULLY CREEK

5. BROADVIEW

6. DALMENY

7. DILKE

8. EASTEND

9. ENDEAVOUR

10. FORT QU'APPELLE

11. FORT WALSH

12. GARDINER DAM

13. GLAMIS-WISETON

14. GOODSOIL

15. GOVENLOCK

16. HAFFORD

17. HARRIS

18. HERBERT

19. HUMBOLDT

20. INDIAN HEAD

21. KELVINGTON

22. KENASTON

23. KILLSQUAW LAKE

24. KINDERSLEY

25. KUTAWAGAN LAKE

26. LA RONGE

27. LAST MOUNTAIN LAKE
28. LOON LAKE

29. LUSELAND

30. MAIDSTONE BRIDGE

31. MELFORT

32. MOOSE JAW

33. PRINCE ALBERT

34. PRINCE ALBERT

35. PRINCE ALBERT

36. RAYMORE

37. REGINA

38. ROUND LAKE

39. ROUND LAKE

40. SASKATCHEWAN FORKS

41. SASKATOON

42. SCOTT

43. SEMANS

44. SKULL CREEK

45. SOMME

46. SPIRIT LAKE

47. SPRING VALLEY

48. SQUAW RAPIDS

49. TISDALE

50. WAUCHOPE

51. WEBB

52. WHITE BEAR

53. WHITEBEECH

54. WOLSELEY

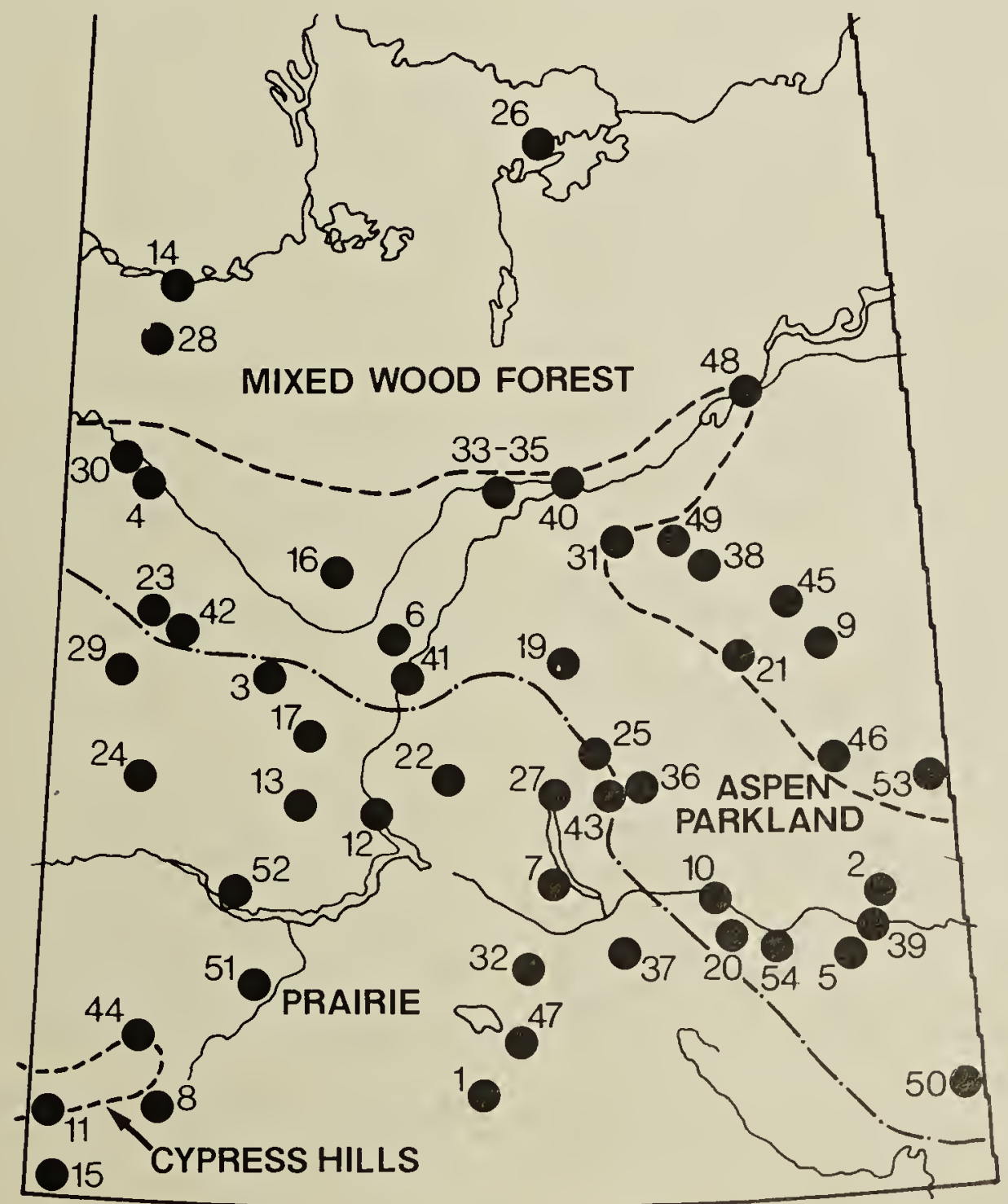




\section{Count Areas and Participants}

1. ASSINIBOIA. Gordon Brunt, Jack Burgeson, Alex Duxbury, Cecil T. Hayward (compiler), Donald Lemond, Don Lewis, Ted McMorrine, Wilf Prentice, Ken Schuweiler.

2. BANGOR. Katie Thompson.

3. BIGGAR. Harvey Renaud, Wayne Renaud, Guy Wapple (compiler), Marguerite Wapple, Robert Wapple.

4. BIG GULLY CREEK. Wayne Harris (compiler), Sheila Lamont.

5. BROADVIEW. Dave J. Chaskavich.

6. DALMENY. Lorne, Brian, Gilbert, Ann and Mara Sperling, Lloyd Sperling (compiler).

7. DILKE. Margaret Belcher (compiler), Doug Laing.

8. EASTEND. Tom Donald, Mel Fitch, Jane Gollop, Mike Gollop (compiler), Barbara Shourounis.

9. ENDEAVOUR. Norman Harris.

10. FORT QU'APPELLE. Manley Callin (compiler), Errol Cochrane, Ethel Cockwill, Bernie and Ethel deVries, Flossie Fowler, Peter Guzak, Alma Hiebert, Ronald Hooper, Lois Lamontagne, Alice Laing, Fern McDougall, Alan Mlazgar, John Norman, Lorne and Doreen Rowell, Kay Ruud, Dorothy Williams.

11. FORT WALSH. Bob and Dixie Black, Nigel Caulkett, Chris Escott, Wayne Harris, Ron Jensen, Sheila Lamont, G. Wilkes Parsonage, Don Renaud, Wayne Renaud, Stan Shadick, Guy Wapple (compiler), Robert Wapple, Jack Wilkinson.

12. GARDINER DAM. Wayne Harris, Sheila Lamont, Wayne Renaud, Guy Wapple (compiler), Robert Wapple.

13. GLAMIS-WISETON. Brian Hones, G.L.Jones (compiler).
14. GOODSOIL. David Morton.

15. GOVENLOCK. Wayne Harris (compiler), Sheila Lamont, Wayne Renaud, Guy Wapple, Robert Wapple.

16. HAFFORD. Terry Toews, Don Weidl (compiler).

17. HARRIS. Guy Wapple (compiler), Robert Wapple.

18. HERBERT. Terry Toews, Don Weidl (compiler).

19. HUMBOLDT. Ed Brockmeyer (compiler), Dwayne Saretsky.

20. INDIAN HEAD. Cec and Betty Ashmore, Marg Barclay, Peter Barrett, Yvonne Brown, Hazel and Ernie Bugloss, Richard Escott, Joan Halford, Gord Howe, Marcella Horsman, Roy and Rose McLaughlin, Doris Pals, Marj Quigley, T. Runge, Lorne and Joan Scott, Reg and Gertie Scott, Fred Skinner, Mary Skinner (compiler), Chas Thompson, Ron Thompson family.

21. KELVINGTON. James Donovan (compiler), Chris Donovan.

22. KENASTON. Margaret, Doug, JoAnn and Lori Beckie, Lawrence Beckie (compiler).

23. KILLSQUAW LAKE. Allan $R$. Smith.

24. KINDERSLEY. Jean Harris (compiler), Keith Harris.

25. KUTAWAGAN LAKE. Wayne Harris (compiler), Sheila Lamont.

26. LA RONGE. Ron. Jensen (compiler), Jim Pauil.

27. LAST MOUNTAIN LAKE. Wayne Harris (compiler), Sheila Lamont.

28. LOON LAKE. Frank Scott (compiler), Penny Scott.

29. LUSELAND. E.J. Finley, Estelle Finley, Kim Finley, K.J. Finley (compiler). 
30. MAIDSTONE BRIDGE. Wayne Harris (compiler), Sheila Lamont, Joe Marchand.

31. MELFORT. Genevieve Belliveau, Philip Curry (compiler), Laura Smith, Tom Smith.

32. MOOSE JAW. Carl Ellis, Doug Francis, Edith Kern, Pat Kern, Cy Knight, Leith Knight (compiler), Bernice Lewis, Moray Lewis, Connie McIntyre, Henny and Arie Van Dorland, Gus Zado.

33. PRINCE ALBERT. Ansgar and Christine Aschim, Kevin Aschim (compiler), Kim Hruska, H.A. Martinson.

34. PRINCE ALBERT. Carman Dodge.

35. PRINCE ALBERT. Murray J. Kyle.

36. RAYMORE. Greta Harris, Wayne Harris (compiler), Sheila Lamont.

37. REGINA. Chris Adam, Jessie Bailey, Keith Barr, Margaret Belcher, Tom Beveridge, Frank Brazier, Walter Chapman, Eric Cooke, Jim Hines, Gayl Hipperson, John Jackson, Jim Jowsey, Elizabeth Kassian, Darlene Kauk, Greg Kraetzig, Robert Kreba (compiler), Mr. Kos, Tony Lang, George Ledingham, Christine MacDonald, John MacDonald, Brad Muir, Brian Rainey, Sarogina Ramnarina, Tom Riffel, Anneka Rummens, Helena Rummens, Bill Russon, Diane Secoy, Allan Smith, Frank Switzer, Ian Switzer, Chris Wilhelm, Janie Wilhelm, Pierre Wilhelm, Rita Wilhelm.

38. ROUND LAKE (South of Porcupine Plain). James Donovan.

39. ROUND LAKE (Qu'Appelle Valley). Doug Francis (compiler), Joe Grimeau, Pat Kern.

40. SASKATCHEWAN FORKS (North of Weldon). Kevin Aschim (compiler), Kim Hruska.

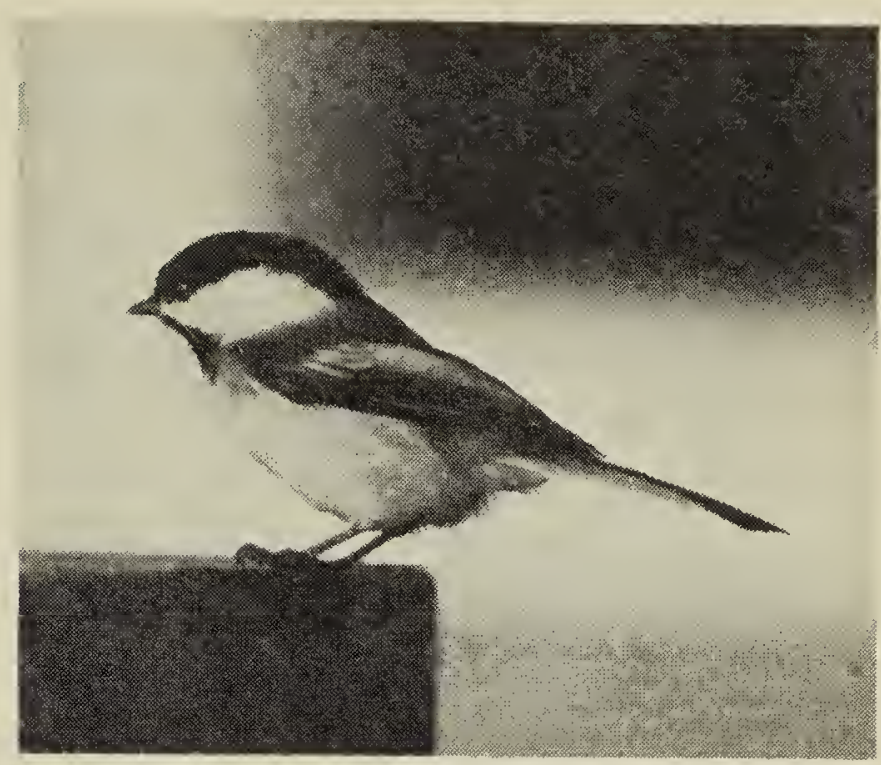

Black-capped Chickadee

41. SASKATOON. Juhachi Asai, Bob, Garth and Joyce Besant, Muriel Carlson, Nigel Caulkett, Chris Escott, Hartley Fredeen, Geoff Galloway, Mary Gilliland, Bernie and Madeleine Gollop, Bruce and John Hanbidge, Simon Heath, David, Mary, and Stuart Houston (compiler), Catherine Laratte, Donna Malcolm, Blake Maybank, Don and Jo McRobbie, Sean Morrissey, Betty and Jim Mundy, Dan Neves, Pat and Jim, O'Neil, Lynn Oliphant, John Polson, Charlie Simpson, Jim Slimmon, Helen and Larry Smith, Jeff Stewart, Judy Taylor, Jim and Shirley Wedgwood, David Wright, Kelly Wylie.

42. SCOTT. Guy Wapple (compiler), Robert Wapple.

43. SEMANS. J. Rawdon Bieber, Tony Lang, Barrie Peeke, Lloyd Saul (compiler), Shabbir Zavery.

44. SKULL CREEK. Jim Bennetto (compiler), Marjorie Mann, Michelle Schuler.

45. SOMME. Stan Back, David Black, Edwin Billeter, Florence Chase, Donald Hooper (compiler).

46. SPIRIT LAKE. Bill Anaka (compiler), Joyce Anaka. 
47. SPRING VALLEY. Allan, Larry and Nick Bogdan, Flossie Bogdan (compiler), Dean Goian.

48. SQUAW RAPIDS. Wayne Harris (compiler), Sheila Lamont, Wayne Renaud, Guy Wapple, Robert Wapple.

49. TISDALE. Joyce Mohr.

50. WAUCHOPE. Sydney Barber, Dale Hjertaas (compiler), Paule Hjertaas.
51. WEBB. Stan Greenwood, Jack Ricou (compiler), Gloria Wright.

52. WHITE BEAR. Darryl, Gary and Laine Jordheim, Sig Jordheim (compiler).

53. WHITEBEECH. Tracy Hutchinson, Ida Wotherspoon, Lindsay Wotherspoon (compiler).

54. WOLSELEY SOUTH. Dale Chay, Donald Hayward (compiler).

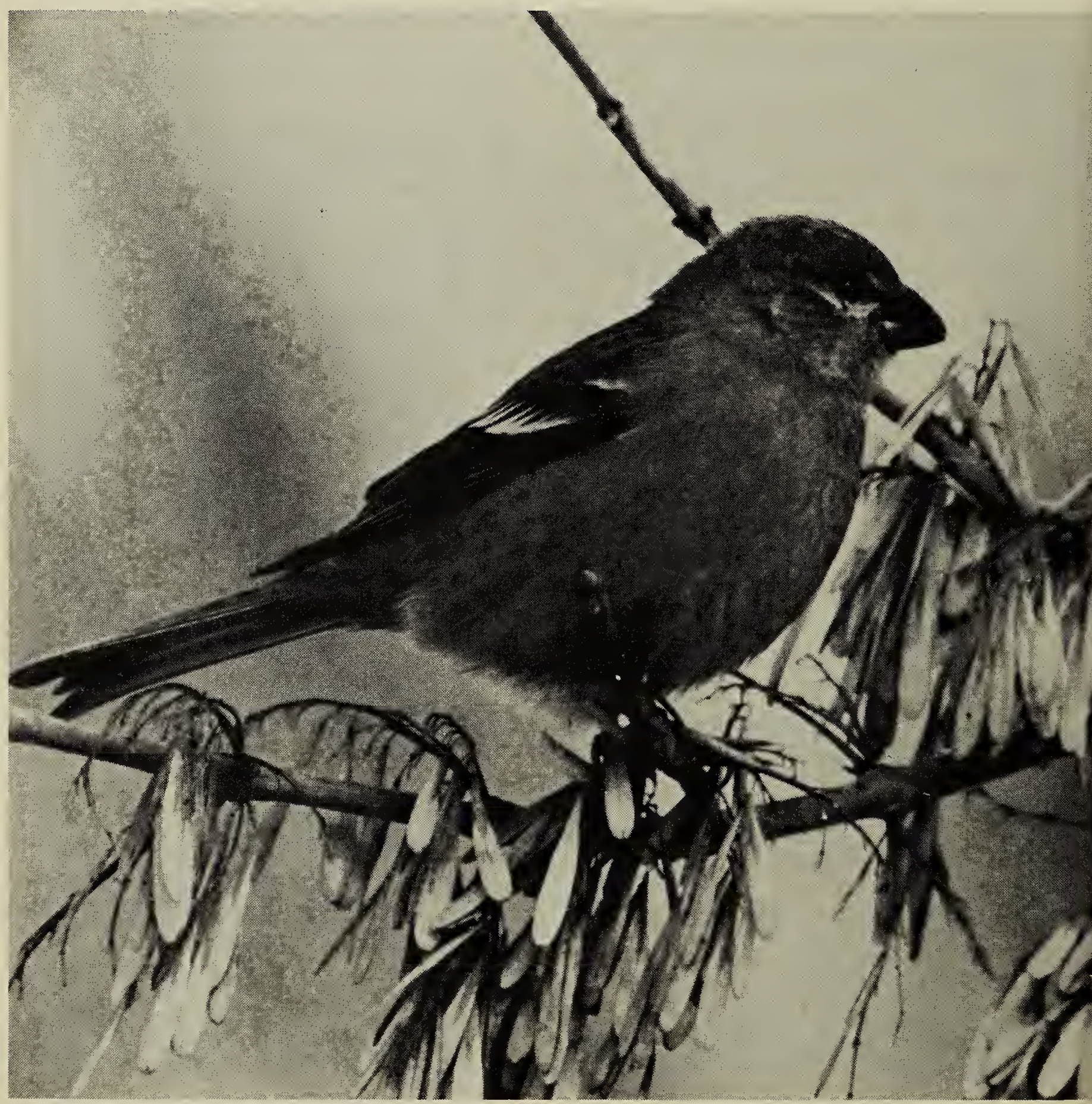

Pine Grosbeak

Fred Lahrman 
Table 1. CHRISTMAS COUNT COVERAGE AND WEATHER CONDITIONS.

\begin{tabular}{|c|c|c|c|c|c|c|c|c|c|}
\hline 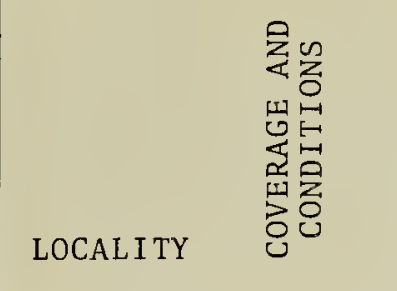 & 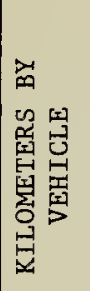 & 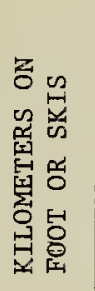 & 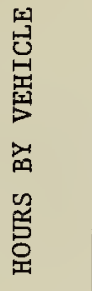 & 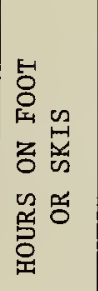 & 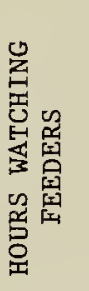 & 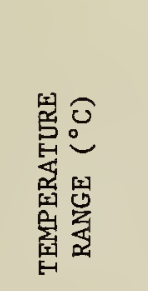 & 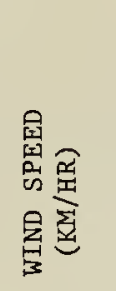 & 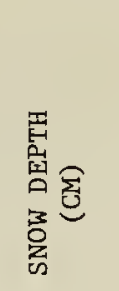 & w \\
\hline ASSINIBOIA & 40 & & 1 & & & -26 & $24-32$ & $20-25$ & partly cloudy \\
\hline BANGOR & 5 & & & & & $-10 /-25$ & 4 & 20 & clear \\
\hline BIGGAR & 268 & 25 & 11 & 9 & & $-10 /-8$ & $5-15$ & $10-40$ & overcast, $11 \mathrm{ght}$ snow \\
\hline BIG GULLY CREEK & 80 & 5 & 3 & 3 & & $-33 /-27$ & $0-10$ & $10-20$ & clear \\
\hline BROADVIEW & 64 & 3 & $3 \frac{1}{2}$ & $1 \frac{1}{2}$ & & $-22 /-24$ & $20-24$ & $10-20$ & partly cloudy \\
\hline DALMENY & & 25 & & 20 & & $-22 /-20$ & $7-10$ & $5-15$ & overcast, clearing \\
\hline DILKE & 81 & 6 & 3 & 2 & & $-37 /-27$ & 5 & $5-30$ & overcast \\
\hline EASTEND & 96 & 1 & $5 \frac{1}{2}$ & $\frac{1}{2}$ & & $-27 /-23$ & 5 & $1-20$ & clear \\
\hline ENDEAVOUR & & 1 & & 3 & & $-36 /-12$ & 0 & $10-12$ & partly cloudy \\
\hline FORT QU'APPELLE & 306 & & 16 & & 3 & -15 & 30 & 15 & clear \\
\hline FORT WALSH & 126 & 45 & 22 & 29 & & $-8 /+2$ & $0-60$ & $10-30$ & partly cloudy \\
\hline GARDINER DAM & 240 & 8 & 13 & $3 \frac{1}{2}$ & & $-8 /-3$ & $40-60$ & $10-40$ & overcast, snow \\
\hline GLAMIS-WISETON & 43 & 3 & 2 & 1 & & $-8 /-12$ & $5-10$ & $5-15$ & clear, becoming cloudy \\
\hline GOODSOIL & 5 & & 1 & & 8 & $-40 /-30$ & $0-5$ & $15-25$ & clear \\
\hline GOVENLOCK & 125 & 13 & 10 & 7 & & $-5 /+3$ & $0-8$ & $0-5$ & clear \\
\hline HAFFORD & 75 & 2 & 3 & 1 & & $-13 /-9$ & $0-5$ & $4-6$ & clear, clouding over \\
\hline HARRIS & 239 & 11 & 6 & 4 & & $-28 /-22$ & $10-0$ & $10-40$ & light snow, clearing \\
\hline HERBERT & 10 & 2 & 1 & 1 & & $-16 /-16$ & $8-8$ & $3-6$ & partly cloudy \\
\hline HUMBOLDT & 30 & 8 & 1 & $1 \frac{1}{2}$ & 2 & $-18 /-25$ & $0-0$ & $8-10$ & clear \\
\hline INDIAN HEAD & 65 & 5 & 3 & $2 \frac{1}{2}$ & $1 \frac{1}{2}$ & $-2 / \quad 0$ & $0-25$ & $7-15$ & mostly clear \\
\hline KELVINGTON & 15 & 1 & 2 & $\frac{1}{2}$ & $\frac{1}{2}$ & $-25 /-32$ & $15-20$ & $20-25$ & mostly clear \\
\hline KENASTON & 48 & 6 & 2 & 4 & & -26 & 0 & 38 & clear \\
\hline KILLSQUAW LAKE & 122 & 2 & $4 \frac{1}{2}$ & 2 & & $-3 /-6$ & $15-25$ & 15 & clear, clouding over \\
\hline KINDERSLEY & & 3 & & & & -10 & 5 & 2 & $?$ \\
\hline KUTAWAGAN LAKE & 130 & 2 & 5 & 1 & & $-31 /-23$ & $10-30$ & $10-20$ & clear \\
\hline LA RONGE & 60 & 2 & 2 & 1 & & $-26 /-14$ & $0-6$ & $25-50$ & partly cloudy, 1ight snow \\
\hline LAST MOUNTAIN LAKE & 120 & 2 & 5 & 1 & & $-25 /-20$ & $10-30$ & $5-15$ & clear \\
\hline LOON LAKE & 25 & & $\frac{7}{2}$ & & 5 & $-15 /-10$ & $0-5$ & 25 & light snow, clearing \\
\hline LUSELAND & 88 & 15 & $2 \frac{1}{2}$ & 3 & 1 & $-26 /-22$ & $0-0$ & $2-20$ & clear \\
\hline MAIDSTONE BRIDGE & 85 & 5 & 4 & 5 & 1 & $-37 /-28$ & $5-20$ & $10-20$ & clear \\
\hline MELFORT & 55 & 5 & 4 & 3 & 1 & $-22 /-38$ & $0-8$ & $15-20$ & clouding over \\
\hline MOOSE JAW & 144 & 19 & 7 & 8 & 2 & $-12 /-10$ & 5 & $\frac{1}{2}-2$ & clear \\
\hline PRINCE ALBERT (ASCHIM) & & 16 & & 7 & 3 & +4 & $0-60$ & $0-1$ & overcast, light snow \& rain \\
\hline PRINCE ALBERT (DODGE) & 24 & & 1 & & & $-2 /+2$ & $60-70$ & $0-2$ & overcast, snow \& rain \\
\hline PRINCE ALBERT (KYLE) & & & & & 2 & $-29 /-26$ & $19-19$ & $8-10$ & partly cloudy, light snow \\
\hline RAYMORE & 165 & 15 & 8 & 6 & 1 & $-33 /-21$ & $0-10$ & $6-15$ & clear \\
\hline REGINA & 615 & 54 & 30 & 25 & 2 & $-16 /-17$ & $20-9$ & $10-30$ & partly cloudy, fog \& snow \\
\hline ROUND LAKE & 15 & 5 & $1 \frac{1}{2}$ & $2 \frac{1}{2}$ & & $-15 /-20$ & $20-60$ & $2-5$ & clear, changing to snow \\
\hline ROUND LAKE-QU 'APPELLE V. & 69 & $4 \frac{1}{2}$ & $4 \frac{1}{2}$ & 2 & 2 & $-32 /-22$ & $5-20$ & $10-15$ & cloudy \\
\hline THE SASKATCHEWAN FORKS & & 5 & 2 & 2 & & -27 & 15 & 10 & clear \\
\hline SASKATOON & 536 & 113 & $45 \frac{1}{2}$ & 58 & 12 & $-27 /-14$ & $0-7$ & $5-7$ & overcast, clearing \\
\hline SCOTT & 230 & 12 & 7 & 4 & & $-25 /-20$ & $0-10$ & $10-50$ & clear \\
\hline SEMANS & 120 & 5 & 3 & 2 & & $-21 /-30$ & $6-20$ & $1-35$ & partly cloudy, clearing \\
\hline SKULL CREEK & 20 & 6 & 3 & 2 & & $-18 / 0$ & $0-30$ & $3-5$ & mostly clear \\
\hline SOME & 122 & 3 & 6 & 5 & 12 & $-10 /-8$ & $9-5$ & $23-25$ & overcast \\
\hline SPIRIT LAKE & 92 & 9 & $4 \frac{1}{2}$ & 4 & 1 & $-2 /+4$ & $10-25$ & $0-2$ & mostly clear \\
\hline SPRING VALLEY & 66 & $\frac{1}{2}$ & 3 & $\frac{1}{2}$ & & $-32 /-16$ & $10-5$ & $45-50$ & $?$ \\
\hline SQUAW RAPIDS & 70 & 22 & 9 & 15 & & $-22 /-12$ & $0-8$ & $10-20$ & light fog, clearing \\
\hline TISDALE & 70 & & $1 \frac{1}{2}$ & & & $-10 /-20$ & & $20-25$ & partly cloudy \\
\hline WAUCHOPE & 25 & 26 & 1 & $9 \frac{1}{2}$ & & -5 & 0 & $25-40$ & overcast \\
\hline WEBB & 192 & 4 & $4 \frac{1}{2}$ & $1 \frac{1}{2}$ & & $-3 /-5$ & $30-70$ & $0-20$ & overcast \\
\hline WHITE BEAR & 24 & 45 & $\frac{1}{2}$ & 8 & $\frac{1}{2}$ & $-9 /-21$ & $0-5$ & $4-8$ & clear \\
\hline WHITEBEECH & & 5 & & 2 & 8 & $-22 /-24$ & $1-2$ & $8-9$ & mostly clear \\
\hline WOLSELEY & 55 & 1 & 2 & $\frac{1}{2}$ & & -2 & $25-30$ & $16-21$ & clear \\
\hline & & & & & & & & & \\
\hline
\end{tabular}


Table 2-1. SPECIES RECORDED FROM MORE THAN THREE LOCALITIES DURING COUNT PERIOD. A PLUS SIGN INDICATES A HIGH COUNT FOR A SPECIES SEEN DURING COUNT PERIOD BUT NOT ON COUNT DAY.

\begin{tabular}{|c|c|c|c|c|c|c|c|c|c|c|c|}
\hline 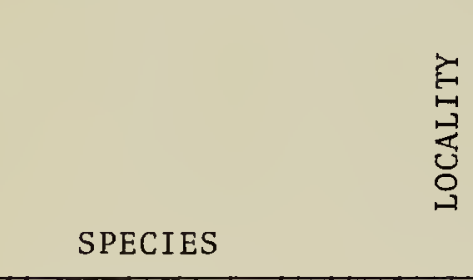 & 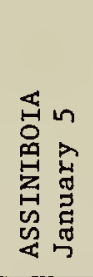 & 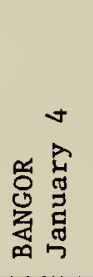 & 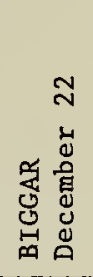 & 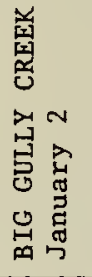 & 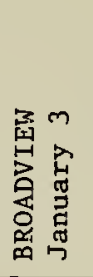 & 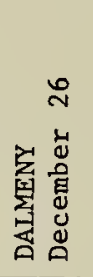 & 量䇰 & 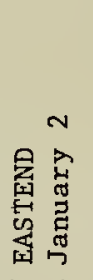 & 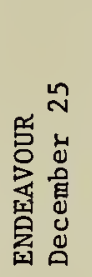 & 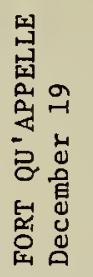 & 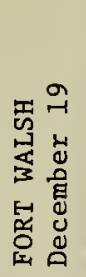 \\
\hline \multirow{5}{*}{$\begin{array}{l}\text { CANADA GOOSE } \\
\text { MALLARD } \\
\text { COMMON MERGANSER } \\
\text { GOSHAWK } \\
\text { RED-TAILED HAWK } \\
\end{array}$} & & & & & & & & & & +3 & \\
\hline & & & & & & & & & & 53 & 1 \\
\hline & & & & & & & & & & +1 & \\
\hline & & & 1 & & & 1 & & 2 & +1 & & 3 \\
\hline & & & & & +1 & & & & & & 1 \\
\hline \multirow{5}{*}{$\begin{array}{l}\text { ROUGH-LEGGED HAWK } \\
\text { GOLDEN EAGLE } \\
\text { BALD EAGLE } \\
\text { PRAIRIE FALCON } \\
\text { MERLIN }\end{array}$} & & & & & & & & & & & \\
\hline & & & & & & & & 1 & & +1 & 2 \\
\hline & & & & & & & & +1 & & & 1 \\
\hline & & & & & & & & & & & 1 \\
\hline & & & 1 & & & & 1 & & & & \\
\hline \multirow{5}{*}{$\begin{array}{l}\text { RUFFED GROUSE } \\
\text { SHARP-TAILED GROUSE } \\
\text { RING-NECKED PHEASANT } \\
\text { GRAY PARTRIDGE } \\
\text { ROCK DOVE } \\
\end{array}$} & & & & & 1 & & & & & 4 & 1 \\
\hline & & & 83 & 5 & 5 & & 24 & & 9 & 3 & 13 \\
\hline & 16 & & & & 1 & & & 2 & & & 14 \\
\hline & 174 & & 93 & & 10 & 7 & 33 & +7 & & 9 & 21 \\
\hline & & & 202 & 1 & 60 & & 44 & 21 & & 104 & 5 \\
\hline \multirow{5}{*}{$\begin{array}{l}\text { GREAT HORNED OWL } \\
\text { SNOWY OWL } \\
\text { SHORT-EARED OWL } \\
\text { HAIRY WOODPECKER } \\
\text { DOWNY WOODPECKER }\end{array}$} & 2 & & 2 & 2 & & 1 & & & & 1 & 7 \\
\hline & 6 & & 1 & & & & +1 & & & 2 & \\
\hline & & & & & & & & +1 & & 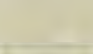 & \\
\hline & & 1 & 2 & 1 & & 1 & & 1 & & 2 & 5 \\
\hline & & 2 & +1 & 3 & & & & & 1 & 2 & 6 \\
\hline \multirow{5}{*}{$\begin{array}{l}\text { HORNED LARK } \\
\text { GRAY JAY } \\
\text { BLUE JAY } \\
\text { BLACK-BILLED MAGPIE } \\
\text { COMRON RAVEN } \\
\end{array}$} & & & 24 & & & & 20 & & 1 & & 13 \\
\hline & & & & & & & & & & & \\
\hline & & & & +1 & 1 & & & & & 6 & 1 \\
\hline & 21 & 1 & 110 & 11 & 9 & 28 & 35 & 114 & 6 & 43 & 230 \\
\hline & & & & & & & & & 20 & & \\
\hline \multirow{5}{*}{$\begin{array}{l}\text { BLACK-CAPPED CHICKADEE } \\
\text { BOREAL CHICKADEE } \\
\text { WHITE-BREASTED NUTHATCH } \\
\text { RED-BREASTED NUTHATCH } \\
\text { BROWN CREEPER }\end{array}$} & & 5 & 20 & 15 & 4. & 7 & 3 & 7 & 2 & 38 & 127 \\
\hline & & & & & & & & & & & \\
\hline & & & & & & & & & & 2 & \\
\hline & & & & & & & & & & & 7 \\
\hline & & & & & & & & +1 & & & 2 \\
\hline \multirow{5}{*}{$\begin{array}{l}\text { AMERICAN ROBIN } \\
\text { GOLDEN-CROWNED KINGLET } \\
\text { BOHEMIAN WAXWING } \\
\text { NORTHERN SHRIKE } \\
\text { STARLING }\end{array}$} & & & & & & & & 9 & & +1 & \\
\hline & & & & & & & & & & & 13 \\
\hline & & 12 & 175 & & 1 & & & 140 & & 91 & 271 \\
\hline & & & & & & & & & & & 2 \\
\hline & & & 3 & & 1 & & & 7 & & & \\
\hline \multirow{5}{*}{$\begin{array}{l}\text { HOUSE SPARROW } \\
\text { RED-WINGED BLACKBIRD } \\
\text { RUSTY BLACKBIRD } \\
\text { EVENING GROSBEAK } \\
\text { PINE GROSBEAK } \\
\end{array}$} & 470 & 6 & 1120 & 70 & 2 & 31 & 190 & 30 & & 236 & 167 \\
\hline & & & 4 & & & & & & & & 7 \\
\hline & & & & & & & & & & & 3 \\
\hline & & 4 & & 13 & 4 & & & 11 & & 24 & \\
\hline & & & & 4 & & & & 2 & 16 & +6 & 2 \\
\hline \multirow{6}{*}{$\begin{array}{l}\text { HOARY REDPOLL } \\
\text { COMMON REDPOLL } \\
\text { RED CROSSBILL } \\
\text { DARK-EYED JUNCO } \\
\text { TREE SPARROW }\end{array}$} & & & & 3 & & & & & & & 3 \\
\hline & & & 1378 & 243 & 280 & 68 & 82 & 310 & 9 & 168 & 68 \\
\hline & & & & & & & & & & & 1 \\
\hline & & & & & & & & & & & 1 \\
\hline & & & & & & & & 2 & & & 16 \\
\hline & & 300 & 315 & 96 & 140 & 83 & 437 & +25 & 25 & 5 & 325 \\
\hline \multirow{3}{*}{$\begin{array}{l}\text { NO. SPECIES IN TABLE } 3 \\
\text { NO. COUNT DAY SPECIES } \\
\text { NO. COUNT PERIOD SPECIES } \\
\text { NO. COUNT DAY INDIVIDUALS }\end{array}$} & & & $\frac{1}{18}$ & & & & & $\frac{1}{15}$ & & 3 & $\frac{3}{36}$ \\
\hline & $\frac{6}{6}$ & $\begin{array}{l}8 \\
8\end{array}$ & $\frac{18}{19}$ & $\frac{13}{14}$ & $\begin{array}{l}14 \\
15\end{array}$ & $\frac{9}{9}$ & $\begin{array}{l}10 \\
11\end{array}$ & $\begin{array}{l}15 \\
21\end{array}$ & $\begin{array}{r}9 \\
10\end{array}$ & $\begin{array}{l}18 \\
26\end{array}$ & $\begin{array}{l}36 \\
36\end{array}$ \\
\hline & 689 & 331 & 3535 & 467 & 519 & 227 & 869 & 659 & 89 & 793 & 1346 \\
\hline
\end{tabular}


Table 2-2. SPECIES RECORDED FROM MORE THAN THREE LOCALITIES DURING COUNT PERIOD. A PLUS SIGN INDICATES A HIGH COUNT FOR A SPECIES SEEN DURING COUNT PERIOD BUT NOT ON COUNT DAY.

\begin{tabular}{|c|c|c|c|c|c|c|c|c|c|c|c|}
\hline 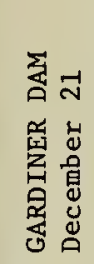 & 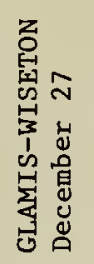 & 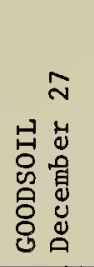 & 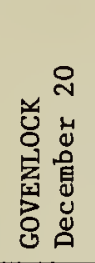 & 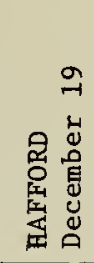 & 窟 & 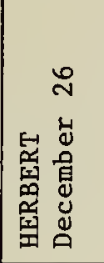 & 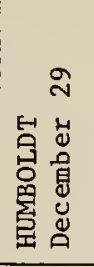 & 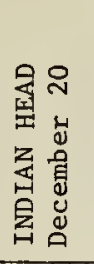 & 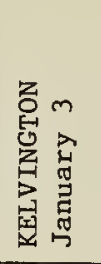 & 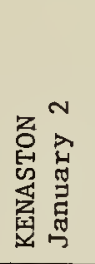 & 䓀 \\
\hline & & & & & & & & +3 & & & \multirow{5}{*}{$\begin{array}{l}\text { CANADA GOOSE } \\
\text { MALLARD } \\
\text { COMMON MERGANSER } \\
\text { GOSHAWK } \\
\text { RED-TAILED HAWK } \\
\end{array}$} \\
\hline 145 & & & & & & & & 7 & & & \\
\hline 2 & & & & & & & & & & & \\
\hline & & 1 & & & & & & 1 & +3 & & \\
\hline & & & & & & & & & & & \\
\hline & & & 1 & & & & & & & & \multirow{5}{*}{$\begin{array}{l}\text { ROUGH-LEGGED HAWK } \\
\text { GOLDEN EAGLE } \\
\text { BAID EAGLE } \\
\text { PRAIRIE FALCON } \\
\text { MERLIN }\end{array}$} \\
\hline & & & 1 & & & & & & & & \\
\hline 7 & & & & & & & & 1 & & & \\
\hline & & & & & & & & & & & \\
\hline & & & & & & & & & & & \\
\hline & & & & & & & & 10 & & & \multirow{5}{*}{$\begin{array}{l}\text { RUFFED GROUSE } \\
\text { SHARP-TAILED GROUSE } \\
\text { RING-NECKED PHEASANT } \\
\text { GRAY PARTRIDGE } \\
\text { ROCK DOVE }\end{array}$} \\
\hline 10 & 27 & 2 & 4 & 1 & 14 & & & 7 & & 4 & \\
\hline & & & 9 & & & & & 2 & & & \\
\hline 57 & 12 & & 11 & & 54 & & +8 & 38 & & 41 & \\
\hline 31 & 100 & & & 117 & 229 & 6 & & 8 & 3 & 27 & \\
\hline 1 & & & 1 & & 1 & 1 & 1 & 3 & & 1 & \multirow{5}{*}{$\begin{array}{l}\text { GREAT HORNED OWL } \\
\text { SNOWY OWL } \\
\text { SHORT-EARED OWL } \\
\text { HAIRY WOODPECKER } \\
\text { DOWNY WOODPECKER }\end{array}$} \\
\hline 1 & 3 & & & & 1 & & +1 & 2 & & 1 & \\
\hline & & & & & & & & & & & \\
\hline & & 1 & & 3 & & & +1 & 5 & 1 & +1 & \\
\hline & & 2 & & 1 & 1 & 1 & & 6 & 1 & +1 & \\
\hline 8 & 3 & & 277 & & 7 & & & & & 5 & \multirow{5}{*}{$\begin{array}{l}\text { HORNED LARK } \\
\text { GRAY JAY } \\
\text { BLUE JAY } \\
\text { BLACK-BILLED MAGPIE } \\
\text { COMMON RAVEN }\end{array}$} \\
\hline & & & & & & & & & & & \\
\hline & & 6 & & 1 & 1 & & & 3 & & & \\
\hline 35 & 15 & 25 & 2 & 17 & 150 & 2 & +10 & 30 & +10 & 15 & \\
\hline & & 5 & & 3 & & & & & 5 & & \\
\hline 4 & & 5 & & 29 & 4 & & 9 & 15 & 4 & +2 & \multirow{5}{*}{$\begin{array}{l}\text { BLACK-CAPPED CHICKADEE } \\
\text { BOREAL CHICKADEE } \\
\text { WHITE-BREASTED NUTHATCH } \\
\text { RED-BREASTED NUTHATCH } \\
\text { BROWN CREEPER }\end{array}$} \\
\hline & & 5 & & & & & & & & & \\
\hline & & 1 & & & & & & 1 & & & \\
\hline & & & & & & & & 5 & & & \\
\hline & & & & & & & & +1 & & & \\
\hline & & & & & & & & & & & \multirow{5}{*}{$\begin{array}{l}\text { AMERICAN ROBIN } \\
\text { GOLDEN-CROWNED KINGLET } \\
\text { BOHEMIAN WAXWING } \\
\text { NORTHERN SHRIKE } \\
\text { STARLING } \\
\end{array}$} \\
\hline & & & & & & & & & & & \\
\hline 139 & & & & 15 & 81 & 35 & & +50 & +46 & +12 & \\
\hline & & & & & & & & & & & \\
\hline & & & & & 3 & & & & +2 & 3 & \\
\hline 311 & 100 & & 361. & 95 & 835 & 70 & 275 & 600 & 70 & 168 & \multirow{5}{*}{$\begin{array}{l}\text { HOUSE SPARROW } \\
\text { RED-WINGED BLACKBIRD } \\
\text { RUSTY BLACKBIRD } \\
\text { EVENING GROSBEAK } \\
\text { PINE GROSBEAK } \\
\end{array}$} \\
\hline & & & 1 & & & & & 1 & & & \\
\hline & & & 3 & & & & & & & & \\
\hline & & 20 & & & & & & & 130 & & \\
\hline 2 & & 1 & & 1 & & & & & 5 & +1 & \\
\hline & & & 1 & & 1 & & & & & & \multirow{5}{*}{$\begin{array}{l}\text { HOARY REDPOLL } \\
\text { COMMON REDPOLL } \\
\text { RED CROSSBILL } \\
\text { DARK-EYED JUNCO } \\
\text { TREE SPARROW }\end{array}$} \\
\hline 179 & & & 465 & 784 & 179 & 1 & 48 & 8 & 2 & 526 & \\
\hline & & & & & & & & 2 & & & \\
\hline & & & & & & & & & & & \\
\hline & & & 3 & & & & & & & & \\
\hline 240 & 70 & +25 & 527 & 24 & 40 & & +282 & 224 & 250 & 34 & SNOW BUNTING \\
\hline 4 & & & 2 & 1 & & & 1 & 1 & & & \multirow{4}{*}{$\begin{array}{l}\text { NO. SPECIES IN TABLE } 3 \\
\text { NO. COUNT DAY SPECIES } \\
\text { NO. COUNT PFRIOD SPECIES } \\
\text { NO. COUNT DAY INDIVIDUALS }\end{array}$} \\
\hline 20 & 8 & 12 & 17 & 14 & 16 & 7 & 4 & 23 & 10 & 11 & \\
\hline 20 & 8 & 13 & 17 & 14 & 16 & 7 & 10 & 26 & 14 & 16 & \\
\hline 1188 & 330 & 74 & 1676 & 1092 & 1601 & 116 & 333 & 980 & 471 & 825 & \\
\hline
\end{tabular}


Table 2-3. SPECIES RECORDED FROM MORE THAN THREE LOCALITIES DURING COUNT PERIOD. A PLUS SIGN INDICATES A HIGH COUNT FOR A SPECIES SEEN DURING COUNT PERIOD BUT NOT ON COUNT DAY.

\begin{tabular}{|c|c|c|c|c|c|c|c|c|c|c|c|}
\hline 鴶 & 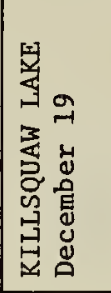 & 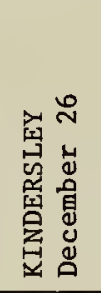 & 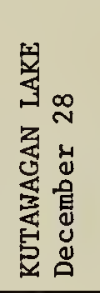 & 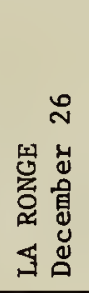 & 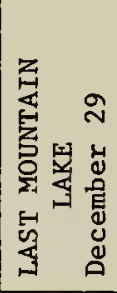 & 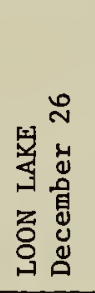 & 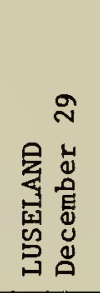 & 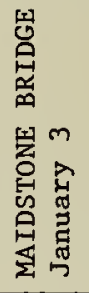 & 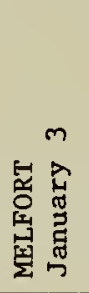 & 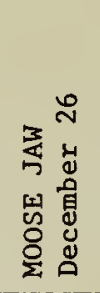 & 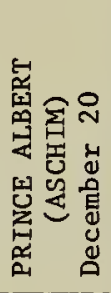 \\
\hline \multicolumn{12}{|l|}{ CANADA GOOSE } \\
\hline \multirow{4}{*}{$\begin{array}{l}\text { MALLARD } \\
\text { COMMON MERGANSER } \\
\text { GOSHAWK } \\
\text { RED-TAILED HAWK }\end{array}$} & 4 & & & & & & & & & & 1 \\
\hline & & & & & & & & & & & \\
\hline & & & & & 1 & & & +1 & & & \\
\hline & & & & & & & & & & & \\
\hline \multirow{5}{*}{$\begin{array}{l}\text { ROUGH-LEGGED HAWK } \\
\text { GOLDEN EAGLE } \\
\text { BALD EAGLE } \\
\text { PRAIRIE FALCON } \\
\text { MERLIN }\end{array}$} & & & & & 1 & & & & & 1 & \\
\hline & & & & & & & & & & & \\
\hline & & & & & & & & & & & \\
\hline & & & & & & & & & & 1 & \\
\hline & & & & & & & & & 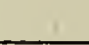 & & \\
\hline \multirow{5}{*}{$\begin{array}{l}\text { RUFFED GROUSE } \\
\text { SHARP-TAILED GROUSE } \\
\text { RING-NECKED PHEASANT } \\
\text { GRAY PARTRIDGE } \\
\text { ROCK DOVE }\end{array}$} & & & & & & & & 1 & 4 & & 1 \\
\hline & & & & 1 & 1 & & 8 & 6 & 1 & & \\
\hline & & & & & & & & & & 16 & \\
\hline & 4 & & 17 & & 50 & & 33 & +6 & 8 & 78 & \\
\hline & 58 & & & & 12 & & 4 & & & 800 & 12 \\
\hline \multirow{5}{*}{$\begin{array}{l}\text { GREAT HORNED OWL } \\
\text { SNOWY OWL } \\
\text { SHORT-EARED OWL } \\
\text { HAIRY WOODPECKER } \\
\text { DOWNY WOODPECKER }\end{array}$} & 1 & & 1 & & 2. & 2 & 1 & 1 & & 1 & 1 \\
\hline & & 1 & 2 & & & +1 & 2 & & & 21 & \\
\hline & & & & & 4 & & & & & 2 & \\
\hline & & & 1 & 2 & & & 1 & 2 & +1 & 2 & 2 \\
\hline & & & & 1 & & 1 & 1 & 1 & +1 & 3 & 3 \\
\hline \multirow{5}{*}{$\begin{array}{l}\text { HORNED LARK } \\
\text { GRAY JAY } \\
\text { BLUE JAY } \\
\text { BLACK-BILLED MAGPIE } \\
\text { COMMON RAVEN }\end{array}$} & & 5 & 1 & & 3 & & & 1 & & & \\
\hline & & & & 8 & & & & & & & 2 \\
\hline & & & & & & 1 & 2 & 4 & 1 & & 3 \\
\hline & 5 & & 27 & & 35 & 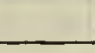 & 20 & 7 & 3 & 40 & 10 \\
\hline & & & & 40 & & 12 & & 2 & 1 & & 23 \\
\hline \multirow{5}{*}{$\begin{array}{l}\text { BLACK-CAPPED CHICKADEE } \\
\text { BOREAL CHICKADEE } \\
\text { WHITE-BREASTED NUTHATCH } \\
\text { RED-BREASTED NUTHATCH } \\
\text { BROWN-CREEPER }\end{array}$} & 2 & & & 14 & & 12 & 1 & 4 & 1 & 19 & 26 \\
\hline & 2 & & & 9 & & & & & & & 3 \\
\hline & & & & & & & & & & 2 & 2 \\
\hline & & & & & & & & & & 1 & \\
\hline & & & & & & & & & & & \\
\hline \multirow{5}{*}{$\begin{array}{l}\text { AMERICAN ROBIN } \\
\text { GOLDEN-CROWNED KINGLET } \\
\text { BOHEMIAN WAXWING } \\
\text { NORTHERN SHIRKE } \\
\text { STARLING }\end{array}$} & & & & & & & & & & 4 & \\
\hline & & & & & & & & & & 3 & \\
\hline & 50 & +30 & & & & & 75 & 8 & 10 & 15 & 10 \\
\hline & & & & & & & & +1 & & & \\
\hline & & & & & 1 & & 6 & & & 191 & \\
\hline \multirow{5}{*}{$\begin{array}{l}\text { HOUSE SPARROW } \\
\text { RED-WINGED BLACKBIRD } \\
\text { RUSTY BLACKBIRD } \\
\text { EVENING GROSBEAK } \\
\text { PINE GROSBEAK }\end{array}$} & 38 & 25 & 120 & & 433 & 50 & 450 & 120 & 197 & 5620 & 34 \\
\hline & & & & & & & & & & & \\
\hline & & & & & & & & & & & \\
\hline & & & & & & 40 & & 15 & & & 155 \\
\hline & & & & & & & & 4 & & & 12 \\
\hline \multirow{6}{*}{$\begin{array}{l}\text { HOARY REDPOLL } \\
\text { COMMON REDPOLL } \\
\text { RED CROSSBILL } \\
\text { DARK-EYED JUNCO } \\
\text { TREE SPARROW }\end{array}$} & & & 7 & & & & & & & & \\
\hline & 465 & 2 & 250 & 20 & 953 & & 225 & 131 & 3 & 83 & \\
\hline & & & & & & & & & & 20 & 2 \\
\hline & & & & & & & & & & 1 & 1 \\
\hline & & & & & & & & & & & \\
\hline & & & 61 & & 7453 & & 265 & 160 & 18 & 260 & \\
\hline NO. SPECIES IN TABLE 3 & 3 & & & 1 & & & & 1 & & 2 & 1 \\
\hline NO. COUNT DAY SPECIES & 13 & 4 & 10 & 9 & 13 & 7 & 15 & 17. & 11 & 24 & 20 \\
\hline NO. COUNT PERIOD SPECIES & 13 & 5 & 10 & 9 & 13 & 8 & 15 & 20 & 13 & 25 & 20 \\
\hline NO. COUNT DAY INDIVIDUALS & 635 & 33 & 487 & 97 & 8949 & 118 & 1094 & 468 & 247 & 7186 & 310 \\
\hline
\end{tabular}


Table 2-4. SPECIES RECORDED FROM MORE THAN THREE LOCALITIES DURING COUNT PERIOD. A PLUS SIGN INDICATES A HIGH COUNT FOR A SPECIES SEEN DURING COUNT PERIOD BUT NOT ON COUNT DAY.

\begin{tabular}{|c|c|c|c|c|c|c|c|c|c|c|c|}
\hline 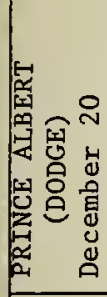 & 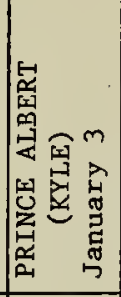 & 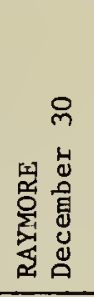 & 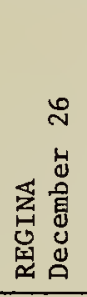 & 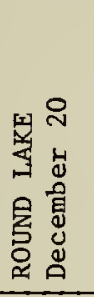 & 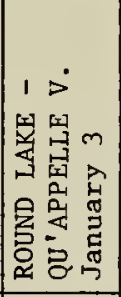 & 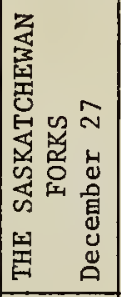 & 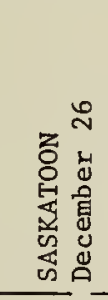 & 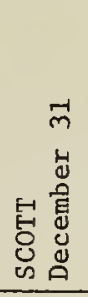 & 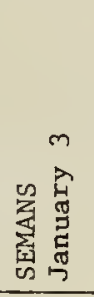 & 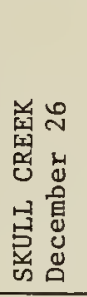 & 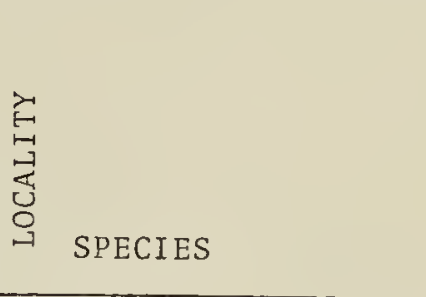 \\
\hline & & & 425 & & 2 & & 1 & & & & \multirow{5}{*}{$\begin{array}{l}\text { CANADA GOOSE } \\
\text { MALLARD } \\
\text { COMMON MERGANSER } \\
\text { GOSHAWK } \\
\text { RED-TAILED HAWK }\end{array}$} \\
\hline & & & 70 & & & & 5 & & & & \\
\hline & & & & & & & 1 & & & & \\
\hline & & 1 & 1 & & & & +1 & & & & \\
\hline & & & & & & & & & & & \\
\hline & & & & & & & & & & & \multirow{5}{*}{$\begin{array}{l}\text { ROUGH-LEGGED HAWK } \\
\text { GOLDEN EAGLE } \\
\text { BALD EAGLE } \\
\text { PRAIRIE FALCON } \\
\text { MERLIN }\end{array}$} \\
\hline & & & & & & & & & & +2 & \\
\hline & & & & & & & & & & & \\
\hline & & & & & & & & & & +1 & \\
\hline & & & 3 & & +1 & & 8 & & & & \\
\hline & & +1 & & 1 & 5 & & 1 & & & & \multirow{5}{*}{$\begin{array}{l}\text { RUFFED GROUSE } \\
\text { SHARP-TAILED GROUSE } \\
\text { RING-NECKED PHEASANT } \\
\text { GRAY PARTRIDGE } \\
\text { ROCK DOVE } \\
\end{array}$} \\
\hline & & 16 & 14 & 6 & 18 & 1 & 68 & 15 & & 1 & \\
\hline & & 2 & & & 5 & & 4 & & 1 & 1 & \\
\hline & & 80 & 261 & & 10 & & 35 & 27 & 5 & 2 & \\
\hline & & 160 & 771 & & T & & 2319 & 47 & 31 & & \\
\hline & & 17 & 3 & & 3 & & 1 & 2 & 1 & 5 & \multirow{5}{*}{$\begin{array}{l}\text { GREAT HORNED OWL } \\
\text { SNOWY OWL } \\
\text { SHORT-EARED OWL } \\
\text { HAIRY WOODPECKER } \\
\text { DOWNY WOODPECKER }\end{array}$} \\
\hline & & 1 & 8 & & & & & 5 & +1 & & \\
\hline & & & & & & & & & & & \\
\hline & 2 & 3 & & 3 & 2 & & 12 & & & & \\
\hline & 2 & +1 & +1 & & 2 & & 13 & & & 3 & \\
\hline & & 2 & 1 & & & & & 18 & 4 & 10 & \multirow{5}{*}{$\begin{array}{l}\text { HORNED LARK } \\
\text { GRAY JAY } \\
\text { BLUE JAY } \\
\text { BLACK-BILLED MAGPIE } \\
\text { COMMON RAVEN }\end{array}$} \\
\hline & & & & 3 & & & & & +1 & & \\
\hline & 1 & 2 & & 1 & 1 & 2 & 18 & & & & \\
\hline 3 & 2 & 67 & 34 & 2 & 18 & 7 & 279 & 30 & 6 & 29 & \\
\hline 3 & 1 & & & 19 & & 6 & 1 & & & & \\
\hline 5 & 4 & 12 & 10 & 5 & 14 & 6 & 158 & 4 & & 23 & \multirow{5}{*}{$\begin{array}{l}\text { BLACK-CAPPED CHICKADEE } \\
\text { BOREAL CHICKADEE } \\
\text { WHITE-BREASTED NUTHATCH } \\
\text { RED-BREASTED NUTHATCH } \\
\text { BROWN CREEPER }\end{array}$} \\
\hline & & & & & & & 1 & 2 & & & \\
\hline 2 & 2 & & & & 3 & & 4 & & & & \\
\hline & & & 2 & & & & 12 & & & & \\
\hline & & & 1 & & & & & & & & \\
\hline & & & 2 & & 2 & & 1 & 1 & & & \multirow{5}{*}{$\begin{array}{l}\text { AMERICAN ROBIN } \\
\text { GOLDEN-CROWNED KINGLET } \\
\text { BOHEMIAN WAXWING } \\
\text { NORTHERN SHRIKE } \\
\text { STARLING }\end{array}$} \\
\hline & & & 4 & & & & 1 & & & & \\
\hline & & +250 & 2 & & 200 & & 3614 & 22 & & 229 & \\
\hline & & 1 & & & & & 1 & & & & \\
\hline & & 45 & 209 & & & & 86 & & & & \\
\hline & 50 & 1430 & 3097 & & 25 & & 2589 & 554 & 118 & 50 & \multirow{5}{*}{$\begin{array}{l}\text { HOUSE SPARROW } \\
\text { RED-WINGED BLACKBIRD } \\
\text { RUSTY BLACKBIRD } \\
\text { EVENING GROSBEAK } \\
\text { PINE GROSBEAK }\end{array}$} \\
\hline & & & & & & & & & & & \\
\hline & & 1 & & & & & & & & +6 & \\
\hline & 200 & 6 & & 7 & 50 & & 8 & & & +15 & \\
\hline & & +5 & & & & 3 & 12 & & & & \\
\hline & & 1 & 15 & & 60 & & 52 & 1 & & & \multirow{5}{*}{$\begin{array}{l}\text { HOARY REDPOLL } \\
\text { COMMON REDPOLL } \\
\text { RED CROSSBILL } \\
\text { DARK-EYED JUNCO } \\
\text { IERK SPARROW }\end{array}$} \\
\hline & & 1264 & 515 & & 115 & & 2291 & 331 & 267 & 41 & \\
\hline & & & & & & & & & & & \\
\hline & & & & & & & & & & & \\
\hline & & & & & & & 2 & & & 15 & \\
\hline & & 2608 & 199 & & 30 & 310 & 20 & 255 & 339 & & SNOW BUNTING \\
\hline & & & 6 & & 1 & 1 & 6 & & & & \multirow{4}{*}{$\begin{array}{l}\text { NO. SPECIES IN TABLE } 3 \\
\text { NO. COUNT DAY SPECIES } \\
\text { NO. COUNT PERIOD SPECIES } \\
\text { NO. COUNT DAY INDIVIDUALS }\end{array}$} \\
\hline 4 & 9 & 20 & 26 & 9 & 19 & 8 & 37 & 15 & 9 & 12 & \\
\hline 4 & 9 & 24 & 29 & $?$ & 21 & 8 & 38 & 15 & 11 & 16 & \\
\hline 13 & 264 & 5719 & 5672 & 47 & 565 & 336 & 11723 & 1314 & 772 & 409 & \\
\hline
\end{tabular}


Table 2-5. SPECIES RECORDED FROM MORE THAN THREE LOCALITIES DURING COUNT PERIOD. A PLUS SIGN INDICATES A HIGH COUNT FOR A SPECIES SEEN DURING COUNT PERIOD BUT NOT ON COUNT DAY.

\begin{tabular}{|c|c|c|c|c|c|c|c|c|c|c|c|}
\hline SPECIES & 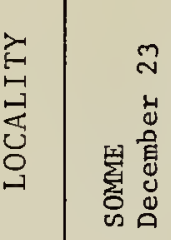 & 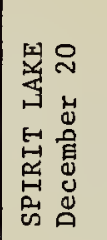 & 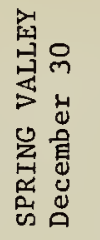 & 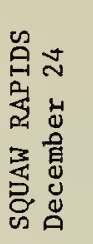 & 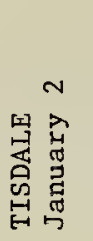 & 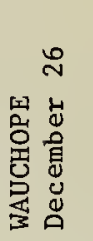 & 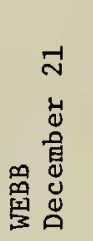 & 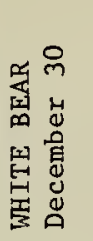 & 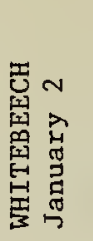 & 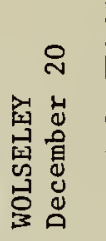 & 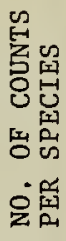 \\
\hline \multirow{5}{*}{$\begin{array}{l}\text { CANADA GOOSE } \\
\text { MALLARD } \\
\text { COMMON MERGANSER } \\
\text { GOSHAWK } \\
\text { RED-TAILED HAWK }\end{array}$} & & & & & & & & & & & 5 \\
\hline & & & & 6 & & & 40 & & & & 10 \\
\hline & & & & 11. & & & & & & & 4 \\
\hline & 2 & +1 & & 10 & & & & +1 & & & 17 \\
\hline & & 1 & & 1 & & & & & & & 4 \\
\hline \multirow{5}{*}{$\begin{array}{l}\text { ROUGH-LEGGED HAWK } \\
\text { GOLDEN EAGLE } \\
\text { BALD EAGLE } \\
\text { PRAIRIE FALCON } \\
\text { MERLIN } \\
\end{array}$} & 2 & 1 & & 2 & & & & & & & 6 \\
\hline & & & +1 & & & & 1 & 1 & & & 8 \\
\hline & +1 & & & 52 & & & & & & & 6 \\
\hline & & & & & & & & +1 & & & 4 \\
\hline & +1 & & & & & & & 1 & t & & 7 \\
\hline \multirow{5}{*}{$\begin{array}{l}\text { RUFFED GROUSE } \\
\text { SHARP-TAILED GROUSE } \\
\text { RING-NECKED PHEASANT } \\
\text { GRAY PARTRIDGE } \\
\text { ROCK DOVE } \\
\end{array}$} & +2 & 3 & & & & 1 & & & +1 & 3 & 16 \\
\hline & 13 & 1 & 16 & 7 & 8 & & 1 & 31 & & 5 & 36 \\
\hline & $\ldots$ & & +2 & & & 1 & & & & . & 14 \\
\hline & 30 & & 81 & & & 29 & & 77 & & 28 & 34 \\
\hline & 40 & & 26 & & & 1 & 94 & 55 & & & 30 \\
\hline \multirow{5}{*}{$\begin{array}{l}\text { GREAT HORNED OWL } \\
\text { SNOWY OWL } \\
\text { SHORT-EARED OWL } \\
\text { HAIRY WOODPECKER } \\
\text { DOWNY WOODPECKER }\end{array}$} & 1 & 3 & 2 & 2 & & 1 & & +1 & & 1 & 35 \\
\hline & +1 & & +2 & 1 & & & & & & & 22 \\
\hline & & & +1 & & & & & & & & 4 \\
\hline & 3 & 7 & & 7 & & 2 & & & 1 & 1 & 31 \\
\hline & 3 & 8 & & 2 & 2 & 5 & & +1 & 2 & & 33 \\
\hline \multirow{5}{*}{$\begin{array}{l}\text { HORNED LARK } \\
\text { GRAY JAY } \\
\text { BLUE JAY } \\
\text { BLACK-BILLED MAGPIE } \\
\text { COMMON RAVEN } \\
\end{array}$} & 1 & & 9 & & & & 16 & 18 & & & 22 \\
\hline & 5 & & & 13 & & & & & & & 6 \\
\hline & 6 & 1. & & 12 & 4 & & & & & & 23 \\
\hline & 18 & 24 & 3 & 27 & 3 & 17 & 12 & 108 & & 3 & 50 \\
\hline & 61 & 1 & & 83 & 4 & & & & +1 & & 19 \\
\hline \multirow{5}{*}{$\begin{array}{l}\text { BLACK-CAPPED CHICKADEE } \\
\text { BOREAL CHICKADEE } \\
\text { WHITE-BREASTED NUTHATCH } \\
\text { RED-BREASTED NUTHATCH } \\
\text { BROWN CREEPER }\end{array}$} & 22 & 22 & & 19 & 3 & 25 & & 2 & 5 & 1 & 44 \\
\hline & 6 & & & 6 & & & & & & & 8 \\
\hline & +1 & 2 & & 1 & & & & & & & 12 \\
\hline & & & & & & & & & & & 5 \\
\hline & +1 & & & & & & & & & & 5 \\
\hline \multirow{5}{*}{$\begin{array}{l}\text { AMERICAN ROBIN } \\
\text { GOLDEN-CROWNED KINGLET }\end{array}$} & & & & & & & & & & & 7 \\
\hline & & & & 1 & & & & & & & 5 \\
\hline & 15 & 8 & & & & & 10 & & +4 & +27 & 31 \\
\hline & +1 & +1 & & 2 & & & & & & & 7 \\
\hline & 1 & 1 & 3 & & & & & 4 & & & 16 \\
\hline \multirow{5}{*}{$\begin{array}{l}\text { HOUSE SPARROW } \\
\text { RED-WINGED BLACKBIRD } \\
\text { RUSTY BLACKBIRD } \\
\text { EVENING GROSBEAK } \\
\text { PINE GROSBEAK } \\
\end{array}$} & 124 & 142 & 262 & 3 & 10 & 486 & 400 & 200 & & 27 & 47 \\
\hline & & & & & & & & & & & 4 \\
\hline & & & & & & & & & & & 4 \\
\hline & 76 & 11 & & 8 & & & & & 10 & & 20 \\
\hline & 26 & 5 & & 36 & & & & & +4 & & 19 \\
\hline \multirow{5}{*}{$\begin{array}{l}\text { HOARY REDPOLL } \\
\text { COMMON REDPOLL } \\
\text { RED CROSSBILL } \\
\text { DARK-EYED JUNCO } \\
\text { TREE SPARROW }\end{array}$} & +5 & 8 & & 3 & & & & & & & 13 \\
\hline & +40 & 104 & +15 & 148 & & 134 & 70 & 41 & 4 & 31 & 43 \\
\hline & & & & & & & & & & & 4 \\
\hline & & +1 & & & & & & & & & 4 \\
\hline & & & & & & & & & & & 5 \\
\hline SNOW BUNTING & 54 & 66 & +900 & 251 & 20 & 97 & 400 & 80 & & 18 & 42 \\
\hline \multirow{4}{*}{$\begin{array}{l}\text { NO. SPECIES IN TABLE } 3 \\
\text { NO. COUNT DAY SPECIES } \\
\text { NO. COUNT PERIOD SPECIES } \\
\text { NO. COUNT DAY INDIVIDUALS } \\
\end{array}$} & 2 & 1 & & 10 & & & & 1 & & & \\
\hline & 22 & 21 & 8 & 36 & 8 & 12 & 10 & 12 & 5 & 10 & \\
\hline & 33 & 24 & 14 & 36 & 8 & 12 & 10 & 17 & 9 & 11 & \\
\hline & 510 & 420 & 402 & 828 & 54 & 799 & 1044 & 618 & 22 & 118 & \\
\hline
\end{tabular}




\begin{tabular}{|c|c|}
\hline SPECIES & LOCALITIES AND NUMBERS SEEN \\
\hline WHITE PELICAN & GARDINER DAM, 1 . \\
\hline DOUBLE-CRESTED CORMORANT & SQUAW RAPIDS, 1. \\
\hline WHITSLING SWAW & GARDTNER DAM, 3. \\
\hline GADWALL & KILLSQUAW LAKE, 2. \\
\hline NORTHERN SHOVELER & KILLSQUAW LAKE, 1. \\
\hline LESSER SCAUP & FORT QU'APPELLE, +1; KTLLSQUAW LAKE, 3. \\
\hline COMMON GOLDENEYE & GARDINER DAM, 11; SASKATOON, 21; SQUAW RAPIDS, 97. \\
\hline WHITE-WINGED SCOTER & GARDINER DAM, 1. \\
\hline RED-BREASTED MERGANSER & SQUAW RAP IDS, 2 . \\
\hline COOPER'S HAWK & FORT QU'APPELLE, +1. \\
\hline GRYFALCON & HAFFORD, 1 ; SQUAW RAPIDS, 1 ; WHITE BEAR, +1 . \\
\hline AMERICAN KESTREL & FORT QU'APPELLE, +1 \\
\hline SPRUCE GROUSE & SQUAW RAPIDS, 1. \\
\hline WILLOW PTARMIGAN & LA RONGE, 2 . \\
\hline SAGE GROUSE & EASTEND, + 1 ; GOVENLOCK, 5 . \\
\hline TURKEY & BIGGAR, 1 . \\
\hline COMMON SNIPE & FORT WALSH, 3. \\
\hline BARRED OWL & SQUAW RAPIDS, 1 ; SOMME, +1 . \\
\hline GREAT GRAY OWL & SQUAW RAPIDS, 1. \\
\hline SAW-WHET OWL & MAIDSTONE BRIDGE, 1 , \\
\hline COMMON FLICKER & MOOSE JAW, 2; REGINA, 9; SASKATOON, 2. \\
\hline PILEATED WOODPECKER & SPIRIT LAKE, 1; SQUAW RAPIDS, 1. \\
\hline BLACK-BACKED THREE-TOED WOODPECKER & THE SASKATCHEWAN FORKS, 1. \\
\hline NORTHERN THREE-TOED WOODPECKER & FORT WALSH, 2; SOMME, 1; SQUAW RAPIDS, 2. \\
\hline CEDAR WAXWING & HUMBOLDT, +6; REGINA, 14 ; SASKATOON, 8. \\
\hline LOGGERHEAD SHRIKE & FORT WALSH, 1. \\
\hline COMMON GRACKLE & REGINA, 1. \\
\hline NORTHERN CARDINAL & SASKATOON, 1. \\
\hline PURPLE FINCH & MOOSE JAW, +2 ; REGINA, +1 ; ROUND LAKE-QU'APPELLE VALLEY, +2 . \\
\hline PINE SISKIN & PRINCE ALBERT (ASCHIM), 7; SASKATOON, 70. \\
\hline AMERICAN GOLDF INCH & REGINA, 1. \\
\hline WHITE-WINGED CROSSBILL & SOMME, 6; SQUAW RAPIDS, 7. \\
\hline CHIPPING SPARROW & INDIAN HEAD, (1). \\
\hline WHITE-THROATED SPARROW & SASKATOON, 3. \\
\hline SONG SPARROW & REGINA, +1 . \\
\hline LAPLAND LONGSPUR & GOVENLOCK, 4. \\
\hline
\end{tabular}




\section{TOGO, Dec. 20}

Again, interested people set up feeders within an $11.5 \mathrm{~km}$ radius of Togo and carefully recorded all observations at their feeders from 20 December to 2 January. Their best day was 20 December when the following species were recorded: unidentified hawk, 1; Great Horned Owl, 1; Snowy Owl, 1; Pileated Woodpecker, 1; Hairy Woodpecker, 4; Downy Woodpecker, 3; Blue Jay, 2; Black-billed Magpie, 2; Common Raven, 4; Black-capped Chickadee, 15; Boreal Chickadee, 24; White-breasted Nuthatch, 1; Bohemian Waxwing, 102; House Sparrow, 200+; Evening Grosbeak, 92; Pine Grosbeak, 30; Common Redpoll, 20.

In addition the following species were seen during count period: Ruffed Grouse, Sharp-tailed Grouse, Gray Partridge, Dark-eyed Junco and Snow Bunting.

Observers were: Doris and Bert Franklin, Olga and Vic Hilderman, Gladys and Geo. Izzard, Wanda and Cusie May, Elizabeth and Archie Parker, Mary and Dick Smith, and Donelda and Howard Wilson.

We regret this count was received too late to include with the other Christmas Bird Counts.

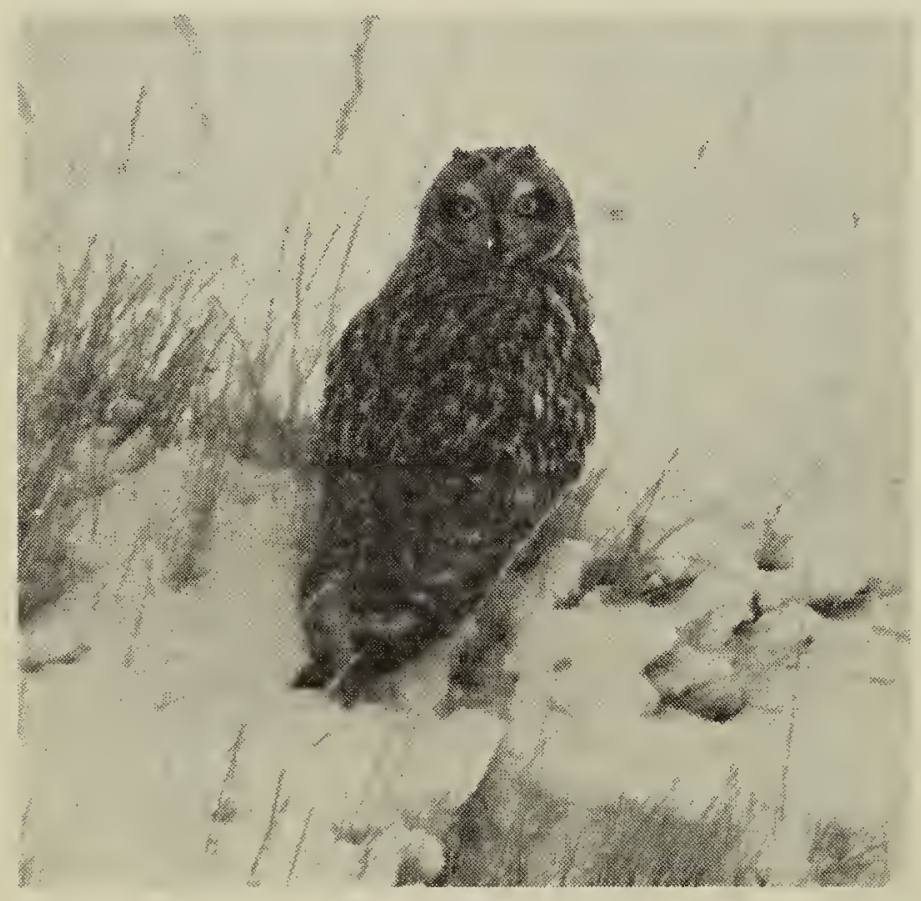

Short-eared Owl
FORT SMITH, NORTHWEST TERRITORIES

DATE: 26 December 1981.

WEATHER: Sunny and clear; temp. $-26^{\circ} \mathrm{C}$; snow $60 \mathrm{~cm}$; daylight hours 0900-1600.

ROUTES COVERED: Fort Smith west along NWT Highway 5 to the Salt River Bridge; Fort Smith south along the Pine Lake road to Salt River; Fort Smith east along the Hay Camp road to Fort Fitzgerald. $140 \mathrm{~km}$ in 3.5 hours.

BIRDS SEEN: Hairy Woodpecker, 2; Gray Jay, 3; Common Raven, 127; Black-capped Chickadee, 2; Boreal Chickadee, 4; Red-breasted Nuthatch, 1; Pine Grosbeak, 42; Hoary Redpoll, 2; Common Redpoll, 8. Nine species, 191 individuals. (Add: Spruce Grouse, 1, Dec. 24; Snowy Owl, 2, Dec. 26.)

CONTRIBUTORS: Don Brannigan, James Darks, Bob Lewis (compiler), Dawna Lewis, Harold Pankratz, Barb Pankratz, Roger Spink, David Splauiski, Shannon Splauiski.

\section{PRAIRIE NEST RECORDS SCHEME}

The Prairie Nest Record Card Scheme requires more participants to record the progress of individual bird nests that are found in Alberta, Manitoba, Saskatchewan and the Northwest Territories. Nest record card data is used by students, federal and provincial government departments, etc., who are doing research on bird species.

Recording cards, information and a report for the 1981 nesting season can be obtained by writing H.W.R. Copland, Prairie Nest Record Card Scheme, Manitoba Museum of Man and Nature, 190 Rupert Avenue, Winnipeg, Manitoba. R3B ON2. 